ISSN 0103-5150

Fisioter. Mov., Curitiba, v. 24, n. 4, p. 621-627, out./dez. 2011

Licenciado sob uma Licença Creative Commons

\title{
Função pulmonar de obesos mórbidos submetidos à cirurgia bariátrica
}

\author{
Pulmonary function of morbidly obese \\ submitted to bariatric surgery
}

\author{
Gabriela Scipioni $^{[a]}$, Fabrício Cieslak ${ }^{[b]}$, Nelson Augusto Rosário Filho ${ }^{[c]}$, Neiva Leite ${ }^{[d]}$ \\ [a] Mestranda em Educação Física na Universidade Federal do Paraná (UFPR), Curitiba, PR - Brasil, e-mail: \\ gabiscipi@yahoo.com.br \\ [b] Doutorando em Educação Física na Universidade Federal do Paraná, (UFPR), Curitiba, PR - Brasil, e-mail: \\ facieslak@gmail.com \\ [c] Doutor da Unidade de Pneumologia na Universidade Federal do Paraná (UFPR), Curitiba, PR - Brasil. \\ [d] Doutora do Programa de Pós-Graduação em Educação Física na Universidade Federal do Paraná (UFPR), \\ Curitiba, PR - Brasil, e-mail: neivaleite@gmail.com
}

\section{Resumo}

Objetivo: Comparar a função pulmonar de indivíduos obesos mórbidos submetidos à gastroplastia. Métodos: Participaram da pesquisa 25 indivíduos, divididos em grupo experimental com 15 sujeitos (feminino = 13 e masculino $=2$ ) e grupo controle com 10 sujeitos (feminino $=8$ e masculino $=2$ ). Foram mensurados os valores espirométricos na fase pré-operatória e na fase pós-operatória, três meses após a cirurgia. Resultados: Verificou-se diferença significante intragrupo na análise inicial e após os três meses de acompanhamento de peso, índice de massa corporal (IMC), Capacidade Vital forçada (CVF), Volume de Reserva Expiratório (VRE), Capacidade Inspiratória (CI) e do Fluxo Expiratório Forçado $\left(\mathrm{FEF}_{25-75 \%}\right)$, nos indivíduos que foram submetidos à cirurgia. Para os resultados obtidos da análise de diferença entre os grupos experimental e controle na análise de grupo e tempo, foram considerados significativos a CVF e o VRE. Verificou-se que aqueles que apresentavam distúrbios inespecíficos na fase pré-operatória passaram a ter função pulmonar normal na fase pós-operatória. Não foram verificadas diferenças significativas entre as características de proporções da espirometria entre os grupos e na avaliação intragrupo experimental. Conclusão: A cirurgia bariátrica influencia na melhora da função pulmonar, observando que um tempo maior de acompanhamento desses 
indivíduos pode nos mostrar resultados ainda mais confirmatórios sobre a importância da perda de peso para a função pulmonar.

Palavras-chave: Obesidade. Volumes pulmonares. Cirurgia bariátrica.

\section{Abstract}

Objective: To compare the pulmonary function of individuals morbidly obese submitted to gastroplasty. Methods: 25 individuals participated of this research, divided in experimental group with 15 subjects (female $=13$ and male $=2$ ) and control group with10 subjects (female $=8$ and male $=2$ ). Spirometric values were measured in the pre-operative and post-operative phase, three months after surgery. Results: There was a significant difference intragroup in the initial analysis after three months follow-up weight, body mass index (BMI), Forced Vital Capacity (FVC), Expiratory Reserve Volume (ERV), Inspiratory Capacity (IC) and the Forced Expiratory Flow $\left(\mathrm{FEF}_{25-75 \%}\right)$, in individuals who underwent surgery. For the results of the analysis of the differences between the experimental and control groups in the analysis of group and time, it were considered significant FVC and ERV. Verified that those nonspecific disturbances in the pre-operative and began to have normal lung function in the post-operative phase. There were not significant differences between the characteristics of the proportions of spirometry between groups and the intragroup experimental evaluation. Conclusion: Bariatric surgery influences in relation to improvement in lung function, observing that a longer follow-up of these individuals can show results in further confirming the importance of weight loss for lung function.

Keywords: Obesity. Lung volumes. Bariatric surgery.

\section{Introdução}

A obesidade mórbida e o acúmulo de gordura resultam em alterações quanto à redução entre os volumes pulmonares, aumentando a resistência do fluxo aéreo e do trabalho respiratório, caracterizando a síndrome da hipoventilação $(1,2)$. 0 presente sintoma acarreta a diminuição capacidade pulmonar total, do volume de reserva expiratório e da capacidade residual funcional (3-5). Podem ocorrer ainda complicações respiratórias como: falta de ar ao mínimo esforço, alteração da mecânica respiratória e apneia durante o sono. Além disso, à medida que o indivíduo se torna mais obeso, há uma sobrecarga muscular para realizar a ventilação, resultando em disfunção da musculatura respiratória $(6,7)$.

Em pesquisas atuais, verificam-se questionamentos relacionados ao efeito da obesidade mórbida sobre a função pulmonar. Os embasamentos referem-se às alterações pulmonares causados por dois aspectos, a dificuldade mecânica ou, se houver, as alterações no metabolismo celular por meio da utilização dos substratos gerados pela obesidade central e pelo acúmulo de tecido adiposo que se localiza na região da cintura (8-10).
Anormalidades da função pulmonar são mais comuns na obesidade central, em que o acúmulo de tecido adiposo localiza-se na região da cintura (10). 0 aumento do índice de massa corporal (IMC) resulta em acréscimo de massa na parede do tórax, como consequência do déficit da mecânica respiratória, resultando em alterações pulmonares restritivas e obstrutivas (11). Não obstante, os valores preditos para mensuração da função pulmonar são designados pelo peso e pela estatura. Nesse sentido, um aumento de tecido adiposo abdominal diminui a excursão do diafragma graças ao aumento do peso e resulta em baixas mensurações de valores preditivos $(12,13)$.

Evidências indicam que a obesidade grau III pode alterar os valores espirométricos, atribuídos ao comprometimento da dinâmica diafragmática e da musculatura da parede torácica. Entretanto, em indivíduos com obesidade grau I e II, essas alterações são muito variáveis e necessitam de avaliações específicas (13). 0 efeito do aumento do IMC em relação às alterações pulmonares ainda não está bem estabelecido em obesos $(14,15)$, de modo que a relação de IMC e doenças respiratórias são aspectos influenciados complicadores como alimentação e baixos níveis de atividade física (15). 
A cirurgia bariátrica demonstra claramente a melhora da relação da obesidade com as condições de comorbidades, incluindo diabetes tipo 2, hipertensão, apneia do sono e dislipidemias (16). No entanto, apesar das evidências sobre as consequências do excesso de peso corporal sobre a função pulmonar em obesos mórbidos, poucos estudos abordaram o impacto da redução do peso sobre a função pulmonar em pacientes com obesidade mórbida $(17,18)$. Nesse sentido, o presente estudo teve como objetivo avaliar as funções pulmonares em indivíduos com obesidade mórbida antes e após a cirurgia de gastroplastia, comparando as possíveis modificações espirométricas com obesos mórbidos que não realizaram a cirurgia bariátrica.

\section{Métodos}

\section{Delineamento do estudo e participantes}

A pesquisa de caráter longitudinal, quase experimental e comparativa foi realizada na cidade de Maringá (Paraná), no ano de 2010. Participaram do estudo 25 indivíduos adultos obesos mórbidos de ambos os sexos, selecionados de forma não aleatória e por conveniência, dispostos no Sistema Único de Saúde (SUS).

Os indivíduos foram divididos em dois grupos: a) Grupo 1 (Experimental): indivíduos submetidos à cirurgia de gastroplastia, acompanhados quanto à função pulmonar nas fases pré e pós-operatória por um período de três meses; e b) Grupo 2 (Controle): indivíduos não submetidos à cirurgia de gastroplastia, acompanhados por um período de três meses. Os sujeitos foram recrutados na Clínica de Obesidade na cidade de Maringá e dispostos em Grupo Experimental (idade média $=40,13+16,75$ anos), que incluiu treze mulheres e dois homens, e Grupo Controle (idade média $=43,50+13,15$ anos), que incluiu oito mulheres e dois homens. Todos os indivíduos obesos mórbidos foram informados sobre os procedimentos utilizados, possíveis benefícios e riscos referentes à execução do estudo. Foram incluídos apenas os participantes que obedeceram aos seguintes critérios: (1) o preenchimento adequado dos critérios de pesquisa; (2) participação voluntária no processo mediante assinatura do termo de consentimento formal.

As coletas foram realizadas nas duas fases, sendo inicial e após três meses. Em cada fase realizou-se uma espirometria na fase pré-operatória na data de internamento do paciente no hospital. Em seguida, aplicaram-se as avaliações de função pulmonar, peso, estatura, índice de massa corporal, pressão arterial sistólica (PAS), pressão arterial diastólica (PAD) e aplicação de um questionário semiestruturado contendo questões referentes à presença de distúrbios pulmonares.

\section{Avaliação espirométrica da função pulmonar}

A função pulmonar foi mensurada por um espirômetro (KoKo-nSpire Health), previamente calibrado com $3 \mathrm{~mL}$, podendo haver mais de uma calibração diária com mudanças bruscas de temperatura, sendo realizada na posição sentada e com o uso de clipe nasal. As variáveis pulmonares mensuradas foram a capacidade vital forçada (CVF) e o volume expiratório forçado no primeiro segundo $\left(\mathrm{VEF}_{1}\right)$, ventilação voluntária máxima (VVM), capacidade vital. Foram realizadas três manobras espirométricas e selecionada aquela com os maiores valores do $\mathrm{VEF}_{1}$ e CVF. Foram apresentados os percentuais dos valores preditos de capacidade inspiratória (CI), pico de fluxo respiratório (PFR), capacidade vital (CV), VVM e VRE conforme protocolo de Crapo (13), conforme protocolo de Pereira (15) para CVF, $\mathrm{VEF}_{1}$, $\mathrm{PEFR}_{\text {e }} \mathrm{FEF}_{25-75 \%}$.

Para o exame de CVF, o indivíduo foi incentivado verbalmente a realizar uma inspiração profunda e posteriormente uma expiração "rápida e prolongada", finalizando o exame com outra inspiração profunda, com a realização de três manobras de CVF, capacidade vital lenta e VVM. Considerou-se a melhor manobra como a função pulmonar de cada fase, sendo que o início e a aceitação do teste foram feitos pela técnica de retroextrapolação, hoje internacionalmente aceita, sendo que o valor desse volume não deve exceder 5\% da CVF (15).

\section{Análise dos dados}

Na descrição dos dados, foram utilizadas medidas paramétricas e não paramétricas. Para as medidas paramétricas, apresentadas em media e desvio-padrão, verificou-se a normalidade dos dados por meio do teste de Shapiro-Wilk; confirmada a ausência de normalidade, utilizou-se o método de transformação de dados para a função logarítmica na base $10\left(\log _{10}\right)$. 
Após a transformação dos dados, aplicou-se a análise de variância multivariada (MANOVA) de medidas repetidas para comparação das variáveis entre os grupos. Em seguida, para análise dos dados não paramétricos, apresentados em percentuais, mediana, valor mínimo e máximo, empregou-se o teste de Friedman de medidas repetidas na análise das variáveis espirométricas entre os grupos, o teste Exato de Fischer para análise das proporções de distúrbios pulmonares entre os grupos e o teste Z para comparações das proporções de medidas repetidas dos valores espirométricos do grupo experimental na fase pré-operatória e pós-operatória. Os procedimentos estatísticos do presente estudo foram realizados mediante a utilização do Statistical Package for the Social Sciences (SPSS, versão 13.0) for Windows, com nível de significância fixado em $\mathrm{p} \leq 0,05$.

\section{Resultados}

Nesse estudo participaram 13 mulheres e 2 homens $(\mathrm{n}=15)$ no grupo experimental, enquanto o grupo controle foi composto de 8 mulheres e dois homens $(\mathrm{n}=10)$, totalizando 25 indivíduos. Na Tabela 1 são apresentadas as variáveis antropométricas e pressóricas iniciais e finais dos grupos. Os dados demonstram diferenças significativas para as variáveis peso e IMC, nas comparações intragrupo experimental e na interação intergrupo e tempo. Para as medidas de pressão arterial, verificou-se diferença significativa apenas na comparação intragrupo controle da PA sistólica.

Na Tabela 2 são apresentadas as variáveis espirométricas iniciais e finais dos grupos. Os dados demonstram diferenças significativas para as variáveis CVF e VRE nas comparações intragrupo experimental e na interação intergrupo e tempo. As variáveis IC e $\mathrm{FEF}_{25-75 \%}$ demonstram diferença significativa apenas na comparação intragrupo experimental.

No Gráfico 1 são apresentados os valores de proporções das ocorrências de doenças pulmonares no grupo experimental $(66,7 \%)$ e no grupo controle (70\%), sendo que a maioria dos pacientes não apresentou histórico de doenças pulmonares, e os outros percentuais foram subdivididos por subgrupos. Não foram verificadas diferenças significativas entre as proporções $\left(X^{2}=5,760 ; p=0,330\right)$.

Os resultados da espirometria na fase pré-operatória demonstraram que entre o grupo controle e experimental houve a presença de distúrbios ventilatórios restritivos, obstrutivos e inespecíficos, este

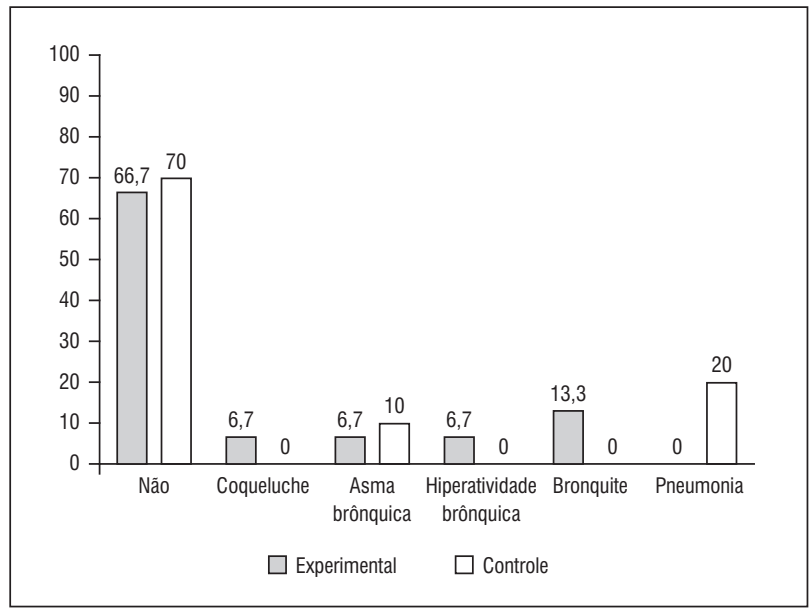

Gráfico 1 - Características iniciais entre os grupos em relação à ocorrência de doenças pulmonares

Fonte: Dados da pesquisa.

Tabela 1 - Médias e desvios-padrões das variáveis antropométricas e pressóricas iniciais e finais dos grupos

\begin{tabular}{|c|c|c|c|c|c|c|c|c|c|c|}
\hline \multirow[b]{2}{*}{ Variável } & \multicolumn{4}{|c|}{ Experimental $(n=15)$} & \multicolumn{4}{|c|}{ Controle $(n=10)$} & \multicolumn{2}{|c|}{ Grupo $\times$ Tempo } \\
\hline & $\begin{array}{c}\text { Inicial } \\
(n=15)\end{array}$ & $\begin{array}{c}\text { Final } \\
(\mathrm{n}=15)\end{array}$ & $\mathbf{F}$ & Valor $\mathbf{p}$ & $\begin{array}{l}\text { Inicial } \\
(\mathrm{n}=10)\end{array}$ & $\begin{array}{c}\text { Final } \\
(n=10)\end{array}$ & $\mathbf{F}$ & Valor $p$ & $F$ & Valor $\mathrm{p}$ \\
\hline Peso & $118,0+21,70$ & $95,8+19,36^{a, b}$ & 342,842 & $<0,001$ & $119,7+27,42$ & $118,9+27,65^{b}$ & 0,945 & 0,356 & 177,048 & $<0,001$ \\
\hline IMC & $44,5+4,69$ & $36,1+4,83^{a, b}$ & 328,469 & $<0,001$ & $46,4+6,53$ & $46,0+6,79^{b}$ & 1,237 & 0,295 & 164,174 & $<0,001$ \\
\hline PA Diastólica & $80,6+4,57$ & $80,6+2,58$ & 0,007 & 0,933 & $87,5+16,54$ & $88,0+22,01$ & 0,010 & 0,922 & 0,020 & 0,888 \\
\hline PA Sistólica & $124,2+14,28$ & $120,6+7,03^{b}$ & 1,716 & 0,211 & $122,6+16,13$ & $131,5+21,86^{a, b}$ & 52,481 & $<0,001$ & 4,788 & 0,039 \\
\hline
\end{tabular}

Legenda: ${ }^{a}=$ Diferença significante inicial e final na análise intragrupo $(p \leq 0,05) ;{ }^{b}=$ Diferença significante na análise grupo $\times$ tempo $(p \leq 0,05)$. Fonte: Dados da pesquisa. 
Tabela 2 - Valores percentuais da função pulmonar entre os grupos

\begin{tabular}{|c|c|c|c|c|c|c|c|c|c|c|}
\hline \multirow[b]{2}{*}{ Variável } & \multicolumn{4}{|c|}{ Experimental $(n=15)$} & \multicolumn{4}{|c|}{ Controle $(n=10)$} & \multicolumn{2}{|c|}{ Grupo $\times$ Tempo } \\
\hline & $\begin{array}{c}\text { Inicial } \\
(n=15)\end{array}$ & $\begin{array}{c}\text { Final } \\
(n=15)\end{array}$ & $F$ & Valor $p$ & $\begin{array}{c}\text { Inicial } \\
(n=10)\end{array}$ & $\begin{array}{c}\text { Final } \\
(n=10)\end{array}$ & $F$ & Valor $\mathrm{p}$ & $\mathbf{F}$ & Valor $\mathrm{p}$ \\
\hline $\mathrm{VEF}_{1}$ & 96 (74-122) & $98(78-123)$ & 3,267 & 0,071 & $87,5(77-116)$ & $90,5(76-114)$ & 0,000 & 1,000 & 2,130 & 0,144 \\
\hline CVF & $95(66-124)$ & $96(75-131)^{a, b}$ & 4,571 & 0,033 & $83,5(73-116)$ & $88(73-114)^{b}$ & 0,143 & 0,705 & 3,857 & 0,050 \\
\hline VRE & $52(11-153)$ & $92(55-155)^{a, b}$ & 8,067 & 0,005 & $37(11-154)$ & $38,5(13-103)^{b}$ & 0,400 & 0,527 & 6,760 & 0,009 \\
\hline $\mathrm{Cl}$ & $111(75-156)$ & $93(82-182)^{a}$ & 7,143 & 0,008 & $108(65-158)$ & $111(80-134)$ & 1,000 & 0,317 & 2,130 & 0,144 \\
\hline PFR & $90(45-117)$ & $75(40-127)$ & 3,267 & 0,071 & $74(57-90)$ & $70,5(51-90)$ & 0,000 & 1,000 & 1,960 & 0,162 \\
\hline IT & $99(92-113)$ & $98(83-114)$ & 1,667 & 0,197 & $103,5(96-138)$ & $103,5(95-113)$ & 1,000 & 0,317 & 2,667 & 0,102 \\
\hline CV & $96(73-122)$ & $95(81-133)$ & 3,267 & 0,071 & $87(78-105)$ & $90,5(72-110)$ & 0,500 & 0,480 & 3,522 & 0,061 \\
\hline VVM & $97(59-129)$ & $91(63-118)$ & 3,267 & 0,071 & $92,5(53-117)$ & $88,5(67-113)$ & 0,400 & 0,527 & 3,240 & 0,072 \\
\hline $\mathrm{FEF}_{25-75 \%}$ & $105(68-147)$ & $88(63-153)^{a}$ & 5,400 & 0,020 & $105,5(74-138)$ & $104(79-140)$ & 0,400 & 0,527 & 1,960 & 0,162 \\
\hline
\end{tabular}

Legenda: ${ }^{\mathrm{a}}=$ Diferença significante inicial e final na análise intragrupo $(\mathrm{p} \leq 0,05) ;{ }^{\mathrm{b}}=$ Diferença significante na análise grupo $\times$ tempo $(\mathrm{p} \leq 0,05)$. Fonte: Dados da pesquisa.

último apenas no grupo experimental (Gráfico 2). Não foram verificadas diferenças significativas entre as proporções $\left(X^{2}=2,315 ; p=0,510\right)$.

Os resultados da espirometria evidenciaram que no grupo experimental houve melhora na função pulmonar no pós-operatório de alguns pacientes (Gráfico 3), embora tenham sido mantidos os mesmos percentuais de pacientes com distúrbio ventilatório restritivo $(6,7 \%)$ e obstrutivo $(6,7 \%)$ na avaliação préoperatória e aos 90 dias de pós-operatório. Verificouse que aqueles que tinham um quadro de distúrbio

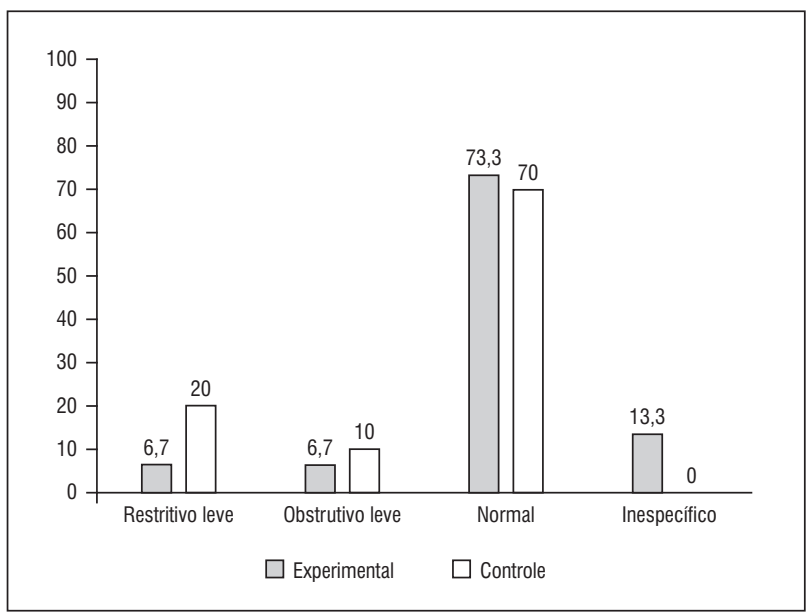

Gráfico 2 - Características iniciais do grupo experimental e controle em relação à espirometria

Fonte: Dados da pesquisa. inespecífico na fase pré-operatória passaram a ter função pulmonar normal na fase pós-operatória. Não foram verificadas diferenças significativas entre as proporções das avaliações pré-operatória e pós-operatória $(\mathrm{Z}=1,414 ; \mathrm{p}=0,157)$.

\section{Discussão}

Ainda são polêmicos os estudos relacionados à obesidade mórbida e suas alterações pulmonares.

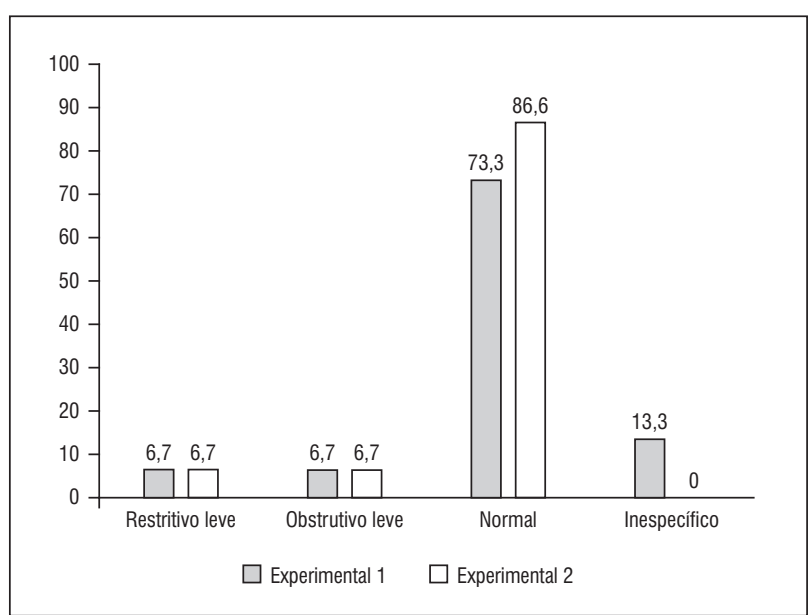

Gráfico 3 - Características iniciais e finais do grupo experimental em relação à espirometria

Fonte: Dados da pesquisa. 
Evidências atuais relatam que a perda de peso por meio da gastroplastia auxilia no controle de distúrbios pulmonares pré-existentes em obesos, prevenindo que indivíduos propensos ao excessivo ganho de peso desenvolvam doenças pulmonares.

Os resultados desse estudo demonstraram redução significativa do IMC e perda de peso em indivíduos submetidos à cirurgia bariátrica. A cirurgia de gastroplastia proposta por Capella auxilia em aproximadamente $40 \%$ da redução do peso corporal após 12 meses, diminuindo o risco de morbidades e comorbidades relacionadas à obesidade $(19,20)$. Em relação à presença dos valores em percentuais encontrados das provas de função pulmonar realizadas nos obesos mórbidos, foi significativo o aumento dos parâmetros avaliados, entre eles, da capacidade vital forçada, do volume de reserva expiratório e do fluxo expiratório forçado no grupo cirúrgico após os três meses de acompanhamento consecutivos, evidenciando que a perda de peso influência na melhora de valores.

A cirurgia de gastroplastia é um dos tratamentos que beneficiam a indivíduos que apresentem distúrbio restritivos ou obstrutivos. Após 12 meses de cirurgia pode haver uma melhora de $12 \%$ em relação a parâmetros de volume expiratório no primeiro segundo $\left(\mathrm{VEF}_{1}\right) ; 9 \%$ da capacidade vital forçada (CVF), possivelmente $15 \%$ de pico de fluxo e $30 \%$ de fluxo expiratório forçado $\left(\mathrm{FEF}_{25-75 \%}\right)$ (21). Após um ano de cirurgia, Maniscalo et al. (22) reportaram que o $\mathrm{VEF}_{1}$ aumentou seu percentil de $83 \%$ para $87 \%$, e a CVF de $75 \%$ para $95 \%$. Dentre outras pesquisas relacionadas, também Marti-Valeri et al. (23) em estudo de 30 indivíduos obteve valores de $\mathrm{VEF}_{1}$ de $78 \%$ para $105 \%$; de CVF de $82 \%$ para $114 \%$ também após um ano de tratamento cirúrgico. São várias as possibilidades da associação da gordura abdominal e efeito no mecanismo da função pulmonar como a limitação da expansibilidade pulmonar levando a distúrbios restritivos, entre eles, o aumento da massa abdominal que impede a excursão diafragmática levando ao aumento da pressão intratorácica (24). Nesta pesquisa houve um aumento da CVF de 95 para $96 \%$ entre a espirometria pré-operatória e após três meses de acompanhamento, e de $52 \%$ para $92 \%$ do VRE, tornando os dados significantes para pesquisa.

Quando perguntados sobre histórico de doença pulmonar, $66,7 \%$ dos indivíduos que se submeteram à cirurgia não apresentaram doença pulmonar, e 70\% do grupo controle relatam também não apresentar, levando a crer que as doenças mais encontradas foram pneumonia (20\%) e bronquite $(13,3 \%)$. Essas doenças podem ter sido desenvolvidas devido à obesidade.

Nos resultados apresentados nos Gráficos 2 e 3 quanto à presença de distúrbios ventilatórios no pré e pós-operatório, foi constatado que indivíduos que apresentavam distúrbios inespecíficos no pré-operatório passaram a ser classificados como indivíduos normais. Resultados previstos por outros autores (25) relatam que a obstrução pulmonar em obesos pode não estar associada a resultados espirométricos, mas, sim, a sintomas de asma e obstrução (25, 26). Para Santana et al. (27) em pesquisa com dois grupos de indivíduos foi verificado que o grupo que apresentava IMC entre $40-59.9 \mathrm{~kg} / \mathrm{m}^{2}$ apresentava $\mathrm{CVF} \mathrm{VEF}_{1}$ e $\mathrm{VEF}_{1} / \mathrm{CVF}$ nos limites da normalidade, enquanto que o outro grupo com IMC $\geq 60$ apresentou $\mathrm{CVF}, \mathrm{VEF}_{1}$ abaixo da normalidade e $\mathrm{VEF}_{1} / \mathrm{CVF}$ ainda dentro dos parâmetros normais, sendo concluído que indivíduos severamente obesos apresentam maior chance de alterações pulmonares.

Há mais de 40 anos se estudam anormalidades na função pulmonar associadas à obesidade. Porém, em estudos atuais ainda se apresenta grande variação sobre a associação de alterações pulmonares com o peso corporal ou mesmo com o índice de massa corpórea (28).

Conclui-se que a cirurgia de gastroplastia apresenta grande relevância no tratamento da obesidade mórbida e que há fortes indícios de que a perda de peso após a cirurgia favorece a melhora das alterações fisiológicas e da mecânica torácica.

\section{Referências}

1. Sharp JT, Henry JP, Sweany SK, Meadows WR, Pietras RJ. The total work of breathing in normal and obese men. J Clin Invest. 1964;43:728-39.

2. Zerah F, Harf A, Perlemuter L, Lorino H, Lorino AM, Atlan G. Effects of obesity on respiratory resistance. Chest. 1993;103(5):1470-6.

3. Lopata M, Onal E. Mass loading, sleep apnea, and the pathogenesis of obesity hypoventilation. 1982;126(4): 640-5.

4. Heinemann F, Budweiser S, Dobroschke J, Pfeifer M. Non-invasive positive pressure ventilation improves lung volumes in the obesity hypoventilation syndrome. Respir Med. 2007;101(6):1229-35. 
5. Ray CS, Sue DY, Bray G, Hansen JE, Wasserman K. Effects of obesity on respiratory function. Am Rev Respir Dis. 1983;128(3):501-6.

6. Wadstrom C, Muller-Suur R, Backman L. Influence of execessive weight loss on respiratory function. A study of obese patients following gastro-plasty. Eur J Surg. 1991;157(5):341-6.

7. Eichenberger A, Proietti S, Wicky S, Frascarolo P, Suter M, Spahn DR, et al. Morbid obesity and postoperative pulmonary atelectasis: an underestimated problem. Anesth Analg. 2002;95(6):1788-92.

8. Collins LC, Hoberty PD, Walker JF, Fletcher EC, Peiris AN. The Effect of body fat distribuition on pulmonary function tests. Chest. 1995;107(5):1298-302.

9. Ray CS, Sue DY, Bray G, Hansen JE, Wasserman K. Effects of obesity on respiratory function. Am Rev Respir Dis. 1983;128(3):501-6.

10. Rigatto AM, Alves SCC, Gonçalves CB, Firmo JF, Provin LM. Performance ventilatória na obesidade. Saúde Rev. 2005;7(17):57-62.

11. Sahbjami H, Gartside PS. Pulmonary function in obese subjects with a normal FEV1/FVC ratio. Chest. 1996; 110(6):1425-9.

12. Crapo RO, Morris AH, Gardner RM. Reference spirometric values using techniques and equipament that meet ATS recommendations. Am Rev Respir Dis. 1981;123(6):659-64.

13. Crapo RO, Morris AH, Clayton PD, Nixon CR. Lung volumes in healthy nonsmoking adults. Bull Eur Physiopathol Respir. 1982;18(3):419-25.

14. Cieslak F, Milano GE, Lopes WA, Radominski RB, Rosário NA Filho, Leite N. O efeito da obesidade sobre parâmetros espirométricos em adolescentes submetidos a broncoprovocação por exercício físico. Acta Scien, Health Scie. 2010;32(1):43-50.

15. Pereira CAC. Espirometria. J. Pneumol. 2002;28(3): 1-82.

16. Guerra S, Sherrill DL, Bobadilla A, Martinez FD, Barbee RA. The relation of body mass index to asthma, chronic bronchitis, and emphysema. Chest. 2002; 122(4);1256-63.

17. Buchwald H, Avidor Y, Braunwald E, Jensen MD, Pories W, Fahrbach K, et al. Bariatric surgery: a systematic revie and meta-analysis. JAMA. 2004;292(14):1724-37.
18. Wadstrom C, Muller-Suur R, Backman L. Influence of execessive weight loss on respiratory function. A study of obese patients following gast. Eur J Surg. 1991; 157(5):341-6.

19. Aaroon SD, Fergusson D, Dent R, Chen Y, Vandemheen $\mathrm{KL}$, Dales RE. Effect of weight reduction on respiratory function and airway reactivity in obese women. Chest. 2004;125(6):2046-52.

20. Kolanowski J. Gastroplasty for morbid obesity: the internist's view. Int J Obes Relat Metab Disord. 1995; 19 Suppl 3:S61-5.

21. Nguyen TN, Hinojosa MW, Smith BR, Gray J, Varela E. Improvement of restrictive and obstructive pulmonary mechanics following laparoscopic bariatric surgery. Surg Endosc. 2009;23(4):808-12.

22. Maniscalo M, Zedda A, Faraone S, Cerbone MR, Cristiano S, Giarddiello C, et al. Weight loss and asthma control in severely obese asthmatic females. Respir Med. 2008;102(1):102-8.

23. Martí-Valeri C, Sabaté A, Masdevall C, Dalmau A. Improvement of associated respiratory problems in morbidly obese patients after open Roux-en-Y gastric bypass. Obes Surg. 2007;17(8):1102-10.

24. Sugerman H, Windsor A, Bessos M, Wolfe L. Intraabdominal pressure, sagittal abdominal diameter and obesity comorbidity. J Intern Med. 1997;241(1):71-9.

25. Sin DD, Jones RL, Man SFP. Obesity is a risk factor for dyspnea but not for airflow obstruction. Arch Intern Med. 2002;162(13):1477-81.

26. Chinn S, Jarvis D, Burney P. Relation of bronchial responsiveness to body mass index in the ECRCHS. Thorax. 2002;57(12):1028-33.

27. Santana AN, Souza R, Martins AP, Macedo F, Rascovski A, Salge JM. The effect of massive weight loss on pulmonary function of morbid obese patients. R Respir Med. 2006;100(6):1100-4.

28. Bedell GN, Wilson WR, Seebohm PM. Pulmonary function in obese person. J Clin Invest. 1958;37(7):1049-60.

Recebido: 05/10/2010

Received: 10/05/2010

Aprovado: 26/05/2011

Approved: 05/26/2011 